\title{
The Influence of Varicocele and Microsurgical Varicocelectomy on Semen Quality, as Determined by Metabolic Analysis
}

\section{Xinzong Zhang}

Guangdong Provincial Family Planning Hospital

\section{Cuncan Deng}

Guangdong Provincial Family Planning Hospital

\section{Wujiang Liu}

Guangdong Provincial Family Planning Hospital

Huang Liu

Guangdong Provincial Family Planning Hospital

\section{Yu Zhou}

Guangdong Provincial Family Planning Hospital

\section{Qianyi Li}

Guangdong Provincial Family Planning Hospital

\section{Houbin Zheng}

Guangdong Provincial Family Planning Hospital

\section{Qiling Wang}

Guangdong Provincial Family Planning Hospital

\section{Min Jiang}

Guangdong Provincial Family Planning Hospital

\section{Tao Pang}

Guangdong Provincial Family Planning Hospital

\section{Chunjie Ma}

Guangdong Provincial Family Planning Hospital

\section{Cheng Huang}

School of Economics and Management, Harbin Institute of Technology, Shenzhen qingguo Zhao

NHC Key Laboratory of Male Reproduction and Genetics, Guangzhou

Yunge Tang ( $\nabla$ tyg813@126.com )

Guangdong Provincial Family Planning Hospital 
Keywords: Varicocele, Microsurgical varicocelectomy, Metabolism, Semen quality

Posted Date: July 15th, 2021

DOI: https://doi.org/10.21203/rs.3.rs-663322/v1

License: (c) (1) This work is licensed under a Creative Commons Attribution 4.0 International License. Read Full License 


\section{Abstract}

The influence of varicocele and microsurgical varicocelectomy on semen quality remains unclear. No previous study has investigated the relationship between semen metabolism and the abnormalities in reproductive function caused by varicocele. Here, we used the non-targeted and targeted metabolic analysis to investigate the different metabolites in seminal plasma within normal, varicocele, and varicocelectomy groups. We clearly showed that varicocele significantly affects sperm metabolism, and microsurgical varicocelectomy can reverse this metabolic abnormality. Moreover, we characterized the landscape of three dipeptides in the seminal plasma of patients with varicocele that have not been identified previously in human tissues or biofluids. Interestingly, the levels of these three dipeptides decreased after microsurgical varicocelectomy coincident with an improvement in semen quality. Western blotting confirmed the downregulation of DPEP3 (dipeptidase 3 ) in the varicocele group and the upregulation of DPEP3 in the varicocelectomy group. Furthermore, we found that eight metabolites may be helpful to distinguish varicocele patients from normal subjects. Our results can be applied to earlier diagnosis or to predict the outcome of microsurgery for varicocele.

\section{Introduction}

Varicocele (VC) is a type of vascular disease that refers to the abnormal expansion, elongation and tortuosity of the veins in the spermatic cord, thus leading to pain, discomfort, and progressive testicular dysfunction. Furthermore, VC is one of the most common causes of male infertility [1]. Previous research reported that the incidence of varicocele ranged from $10-15 \%$ in the male population, and from $19-41 \%$ in infertile males [2]. Varicocele accounts for $30-40 \%$ of cases involving primary male infertility and 69$81 \%$ of cases involving secondary infertility [3]. Due to the lack of conscious symptoms, patients often fail to receive timely diagnosis and treatment; in some patients, this can lead to an impairment of spermatogenesis [4]. Therefore, it is vital that we develop methods to diagnose and treat varicocele in an early manner.

Varicocele has negative effects on sperm concentration, motility, and morphology, and can also affect the integrity of sperm DNA [5]. Over recent years surgical treatment has become the predominant treatment method for varicocele, particularly microsurgical varicocelectomy. Microsurgical varicocelectomy can improve various aspects of sperm quality, including sperm concentration, motility and morphology $[6,7]$. Microsurgical varicocelectomy can also improve sperm retrieval rates in azoospermic patients suffering from varicocele and promote pregnancy and live birth rates in oligospermic patients with varicocele [8]. However, although varicocelectomy can improve sperm quality and clinical outcomes, the precise mechanisms that underlie these effects have yet to be elucidated [9]. Furthermore, the outcome of microsurgical treatment cannot be predicted effectively and despite the application of microsurgery, approximately $30-40 \%$ of patients show no improvements in their sperm parameters[10].

To the best of our knowledge, no previous study has investigated the relationship between sperm metabolism and the abnormalities in reproductive function caused by varicocele. Metabolomics, an 
important research tool for the life sciences is widely used to investigate the clinical diagnosis and pathology of disease [11]. Metabolomics analyses of plasma and blood have also been used to evaluate male infertility [12-13]. Although metabolomics has been widely used to evaluate male infertility, no previous study has attempted to use metabolomics to investigate varicocele. Consequently, we know very little about the metabolic mechanisms responsible for the effects of varicocele on sperm quality or and how varicocelectomy can improve sperm parameters. Furthermore, we do not yet have effective biomarkers for the prediction of such improvements.

In this study, we used ultra-performance liquid chromatography-Q Exactive Orbitrap-Mass spectrometry (UPLC-QE-MS) to carry out untargeted metabolic profiling on seminal plasma. We then analyzed and compared differences in seminal plasma between varicocele patients, the same patients after varicocelectomy, and healthy fertility controls. We compared metabolites across the three groups by correlation analysis, hierarchical clustering analysis, pathways analysis, and network analysis. Potential biomarkers were identified by receiver operating characteristic curve analysis (ROC) and multivariate linear regression. Our aim was to investigate how varicocele affects sperm metabolism and how microsurgery can improve sperm parameters by influencing metabolic events.

\section{Patients And Methods}

Detailed methods can be found in the Supplementary material (Methods section).

\subsection{Patient recruitment}

This study is fully in line with government policies and the Declaration of Helsinki. All experiments were approved by the Medical Ethics Committee of the Family Planning Research Institute of Guangdong Province (Reference: GDJS20200819). The study was registered on the international clinical trial registration website (https://www.researchregistry.com/browse-theregistry\#home/registrationdetails/5ee9edf189de9d0015d6b185/, registration number: researchregistry5714). All patients with varicocele were willing to accept the standard microsurgical varicocele ligation through the inguinal outer ring incision, and signed the informed consent. All volunteers and varicocele patients were willing to donate their semen for the study of varicocele metabonomics. Varicocele patients or paternity-proven controls were screened for eligibility based upon inclusion and exclusion criteria listed in Supplementary Table 1.

\subsection{Semen specimen collection and analysis}

Semen samples were obtained by masturbation (after at least 3 days of abstinence) into a sterile widemouth and metal-free glass container. Samples from the normal group, varicocele group, and varicocelectomy group, were stored at $-80^{\circ} \mathrm{C}$ prior to use. Routine semen analysis was then carried out on a computer-aided semen analyzer (CASA) (WLJY9000, Weili New Century Technology Development Corporation, China), in accordance with guidelines described in the Fifth Edition of the World Health Organization's (WHO) Human Semen Examination Laboratory Manual. 


\subsection{Metabolite Extraction}

A $100 \mu \mathrm{L}$ aliquot of each sample was transferred to a microcentrifuge tube. Then, $400 \mu \mathrm{L}$ of extraction solution (methanol: acetonitrile $=1: 1(\mathrm{v} / \mathrm{v})$, containing isotopic labelled internal standard mixture) was added into each tube, and the sample was vortexed for 30 second. Then, the mixture was sonicated for $10 \mathrm{~min}$ in an ice-water bath and then incubated for 1 hour at $-40^{\circ} \mathrm{C}$ to precipitate proteins. Next, the sample was centrifuged at $12,000 \mathrm{rpm}$ for $15 \mathrm{~min}$ at $4^{\circ} \mathrm{C}$ and the supernatant was transferred to a fresh glass vial for analysis. Quality control (QC) samples were also prepared by mixing equal amounts of supernatant from all samples.

\subsection{LC-MS/MS Analysis}

LC-MS/MS analyses were performed using a UHPLC system (Vanquish, Thermo Fisher Scientific, USA) and a UPLC BEH Amide column $(2.1 \mathrm{~mm} \times 100 \mathrm{~mm}, 1.7 \mu \mathrm{m})$ combined with a Q Exactive HFX mass spectrometer (Orbitrap MS, Thermo). A Thermo Q Exactive HFX mass spectrometer was used to acquire MS/MS spectra in information-dependent acquisition (IDA) mode and was controlled by acquisition software (Xcalibur, Thermo).

\subsection{Data preprocessing and annotation}

First, the original data was converted into mzXML by ProteoWizard software. Then, we used R program package (kernel was XCMS) for peak recognition, extraction, alignment, and integration. Next, metabolite annotation was carried out by matching with an in-house MS database (BiotreeDB V2.1); the cutoff value for annotation was set to 0.3 .

\subsection{Targeted metabolic analysis and western blotting}

Standard tyrosyl-phenylalanine was purchased from Sigma-Aldrich (St. Louis, MO, USA). Standards for tyrosyl-Isoleucine and leucyl-gamma-glutamate were synthesized by GenScript (Nanjing) Co., Ltd. (Nanjing, China). Semen samples from 15 controls with confirmed fertility and 30 varicocele patients including preoperative and postoperative semen were used for DHPLC mass spectrum analysis. The same samples were also used for western blotting to detect DPEP3. RIPA buffer was used to lyse the cells and total protein was extracted. The protein concentration was determined by a BCA Protein Assay Kit ( Thermo Fisher Scientific, Shanghai, China)

. An appropriate amount of each protein sample was denatured and separated by $10 \%$ SDSPAGE(polyacrylamide gel electrophoresis). Separated proteins were then transferred to a Polyvinylidene fluoride (PVDF) membrane, blocked for 1 hour, washed with TBST buffer, and then incubated at $4^{\circ} \mathrm{C}$ overnight with a primary antibody against DPEP3 or GAPDH antibody (1:1,000; Abcam, Cambridge, MA, USA). The following morning, membranes were washed with TBST( Tris Buffered Saline with Tween ${ }^{\circledR}$ 20) buffer, and incubated with a secondary antibody $(1: 2,000)$ at room temperature for 1 hour. The expression levels of DPEP3 were then calculated with GAPDH as internal reference. The experiment was repeated three times independently. 


\subsection{Statistical analysis}

Multidimensional statistical analyses were performed using MetaboAnalyst version 4.0 (http://www.metaboanalyst.ca), and included unsupervised principal component analysis (PCA) and supervised partial least squares discriminant analysis (PLS-DA). Differential metabolites were determined by creating volcano plots with a variable importance (VIP) threshold $>1$ and a $t$-test threshold $<0.05$. Then, we used receiver operating characteristic (ROC) curve analysis to identify differential metabolites and candidate biomarkers. Multiple linear regression analysis was also performed to discover the correlation between different semen parameters (including concentration and motility) and potential biomarkers. SPSS (version 20.0; SPSS IBM Corp., Armonk, NY) was used for all statistical analyses. P values less than 0.05 were regarded as statistically significant.

\section{Results}

\subsection{Clinical characteristics of the participants and outcomes}

The ages and BMI(Body Mass Index) of patients in the fertile control group were matched with those of the varicocele group and the varicocelectomy group $(P>0.05)$. The sperm concentration in the control group $\left(58.30 \pm 22.33 \times 10^{6} / \mathrm{mL}\right)$ was significantly higher than that in the varicocele group $(25.00 \pm 29.81 \times$ $\left.10^{6} / \mathrm{mL}\right)$. The progressive motility of sperm in patients from the varicocele group $(29.09 \% \pm 12.89)$ was significantly lower than that in the control group $(57.97 \% \pm 11.45)$. Both sperm concentration and motility were significantly improved in patients from the varicocelectomy group than the varicocele group $(P<0.05)$. All clinical data are shown in Table 1. 
Table 1

Clinical characteristic of the participants

\begin{tabular}{|c|c|c|c|}
\hline & Normal group & Varicocele group & Varicocelectomy group \\
\hline Number & 30 & 30 & 30 \\
\hline Age & $32.07 \pm 3.93$ & $32.10 \pm 4.15$ & $32.73 \pm 4.14$ \\
\hline BMI & $22.05 \pm 1.91$ & $21.79 \pm 1.88$ & $21.79 \pm 1.88$ \\
\hline Smoking (yes/all) & $0 / 30$ & $0 / 30$ & $0 / 30$ \\
\hline Alcohol (yes/all) & $0 / 30$ & $0 / 30$ & $0 / 30$ \\
\hline Varicocele degree & no & III (only left) & III (only left) \\
\hline Left testicular volume $\left(\mathrm{cm}^{3}\right)$ & $19.79 \pm 3.26$ & $14.18 \pm 1.69$ & $14.18 \pm 1.69$ \\
\hline Right testicular volume $\left(\mathrm{cm}^{3}\right)$ & $20.81 \pm 3.58$ & $15.43 \pm 3.45$ & $15.43 \pm 3.45$ \\
\hline Sperm concentration & $58.30 \pm 22.33$ & $25.00 \pm 29.81^{a}$ & $40.95 \pm 54.04^{b}$ \\
\hline Progressive motility (PR, \%) & $57.97 \pm 11.45$ & $29.09 \pm 12.89^{a}$ & $46.49 \pm 14.96^{b}$ \\
\hline Normal morphological sperm (\%) & $8.41 \pm 3.25$ & $2.00 \pm 1.00^{a}$ & $3.98 \pm 2.38$ \\
\hline DNA fragmentation index & $12.03 \pm 2.96$ & $19.33 \pm 2.75^{a}$ & $14.16 \pm 2.23^{b}$ \\
\hline Serum testosterone(nmol/L) & $19.12 \pm 4.08$ & $14.65 \pm 3.96$ & $16.27 \pm 3.74$ \\
\hline
\end{tabular}

The differential metabolites that were identified by this analysis are shown in Fig. 1(D, E,F) in the form of a volcano plot and shows that there were significant differences in the levels of semen metabolites when compared between the controls, varicocele, and varicocelectomy groups. Compared with the control group, 275 differential metabolites were identified between the varicocele group and the control group. There was also a difference in the levels of semen metabolites after surgery compared to levels before the surgery, but this difference was significantly smaller than when compared to the fertile controls. This suggested that the levels of metabolites may not be fully restored to normal levels following surgery.

\subsection{Hierarchical cluster analysis of differential metabolites}

Figure 2 shows the hierarchical clustering of the top 50 significant different metabolites among the control, varicocele, and varicocelectomy groups. These results showed that there was a certain trend for clustering for several differential metabolites when considered across the three groups. There was also a clustering trend for certain differential metabolites between the varicocelectomy group and varicocele 
groups; however, this difference was significantly smaller than between the varicocele group and the control group.

\subsection{Pathway analysis of different metabolites}

The pathways associated with 275 differentially expressed metabolites between the varicocele group and the control group are shown in Fig. 3(A, B, C). These 275 differential metabolites were associated with 39 pathways in patients with varicocele. Pathway analysis further indicated that varicocele influences semen quality via

taurine and hypotaurine metabolism, amino acid metabolism, glycerol phospholipid metabolism, sphingolipid metabolism, et al. Interestingly, only 17 metabolites were involved in metabolic events involving sphingolipid and glycerophospholipid metabolism, when compared between the varicocelectomy and varicocele groups. This suggested that the improvement of semen quality after microsurgery may be related to the metabolism of glycerophosphate and sphingolipid (Fig. 3A,B,C).

\subsection{Regulatory network analysis for differential metabolites}

After acquiring matching information related to different metabolites, we searched the KEGG database for Homo sapiens (human) and analyzed the network of regulatory interactions. Data arising from this regulatory analysis are shown in a network plot (Fig. 3D,E,F). Although the differences of metabolites between the varicocelectomy and varicocele group were relatively small, the differential metabolites were associated with more complex regulatory networks (Fig. 3), thus implying the importance of these differential metabolites.

\subsection{Potential biomarkers and their correlations with semen parameters}

Next, we performed receiver operating characteristic curve (ROC) analysis for all metabolites. With an area under the curve (AUC) threshold $>0.9$, we identified eight that may represent potential biomarkers, including pyrrolidonecarboxylic acid, cabergoline, 4-hydroxy-2-butenoic acid-gamma-lactone, dimethyl dialkyl ammonium chloride, L-acetylcarnitine, Na-Hexanoyl-Nb-inosityltryptophan, Sodium glycocholate and 5-L-Glutamyl-taurine (Fig. 4 and Supplementary Table 2). Using a threshold of $P<0.05$, no metabolites that were selected by the ROC curves were found to be correlated with sperm concentration. The 5-L-Glutamyl-taurine was significantly positively correlated with motility $(P<0.05)$ while other biomarkers were not correlated with motility. Interestingly, three dipeptides, including leucine-dentalglutamate, tyrosyl-phenylalanine, and tyrosyl-isoleucine, were identified for the first time in human seminal plasma. These were significantly increased in the seminal plasma of patients with varicocele $(P$ $<0.01)$. The quality of semen recovered, and the level of dipeptides, decreased after surgery. These dipeptides represent potential biomarkers for varicocele and useful biomarkers for predicting surgical outcome. The relative abundance of three dipeptides were further confirmed by targeted metabolomics (Fig. 5A). 


\subsection{DPEP3 was downregulated in the varicocele group but upregulated in the varicocelectomy group.}

Next, we used western blotting to compare the levels of DPEP3, a key enzyme involved in testicular dipeptide metabolism, in the normal group, varicocele group, and varicocelectomy group. The relative abundance of DPEP3 was significantly lower in the varicocele group than that in the normal group $(\mathrm{P}<$ $0.01)$ and the varicocelectomy group $(P<0.05)$ (Fig. 5C).

\section{Discussion}

In this study, we first performed UHPLC-QE-MS untargeted metabolomics to detect metabolites in the seminal plasma from controls, varicocele and varicocelectomy groups. Our results clearly show that varicocele significantly affects sperm metabolism, and microsurgical varicocelectomy can reverse this metabolic abnormality. We then identified potential biomarkers to discriminate varicocele from normal controls and to predict the microsurgical outcomes, thus providing tools that can be used to diagnose varicocele earlier. We also identified that certain dipeptides existed in the metabolites of seminal plasma in patient with varicocele; a reduction in the levels of these dipeptides may contribute to the improvement of semen quality after microsurgery.

As showned in Fig. 1A,B,C, the sample quality was good, that the experimental methodology was excellent, and the system stability control was outstanding. Although metabonomics has been applied to the study of male infertility[14-18], so far, it has not been applied to the study of varicocelectomy, especially the change of semen quality after microsurgery. As shown in Fig. 1DEF and Supplementary Table 2, there were marked differences in terms of metabolites in the seminal plasma when compared between the controls, varicocele, and varicocelectomy groups. The reason for this great difference may be that the blood flow is affected by varicocele, resulting in local ischemia and anoxia of testis, and the metabolism of sperm is decreased. Whatever, these results hint that the determination of sperm metabolic changes may be used as a tool for the diagnosis and treatment of varicocele[19]. Our results showed that differences in the levels of metabolites before and after microsurgery were significantly reduced compared with those in patients with varicocele and controls, although there was still strong correlation and clustering properties (Fig. 1). The main reason for these findings may be related to individual genetic background. We compared the same patients before and after surgery; in contrast, patients with varicocele and controls were entirely different individuals.

Our results also showed that the metabolism of glycerophospholipid and sphingolipid were the main changes in sperm metabolism following surgery, and that the main metabolic molecules in these two pathways were significantly up-regulated after microsurgery (Fig. 3). Diphosphatidylglycerol, one of the main phospholipids synthesized by the mitochondrial inner membrane, is related to the assembly and activity of many protein complexes in the mitochondria [20]. These results suggested that the increased mitochondrial activity of sperm may be related to an increase of sperm energy supply and motility. Sphingolipid and its metabolites are not only important structural molecules for the cell membrane, they 
are also involved in many important signal transduction processes, including cell growth, differentiation, senescence, and programmed cell death [21]. This may explain the improvement of sperm morphology (membrane structure), the reduction in sperm apoptosis, and the increase in sperm concentration, following surgery. Although the differences in metabolic molecules before and after microsurgery was relatively small, these differentially expressed molecules were associated with complex molecular network regulation, thus indicating the importance and influence of these differentially expressed molecules (Fig. 3D,E,F).

8 metabolites and 3 dipeptides were identified to be potential biomarkers for varicocele (Figs. 4 and 5). These 3 dipeptides have not been identified in any other biological fluid before. We found that these dipeptides even existed in the subclinical types of varicocele, and can therefore be used as biomarkers for the early diagnosis of varicocele. In patients with varicocele, the concentration of these dipeptides decreased after operation, but still existed. However, the dipeptides were not detectable in the normal group of males. It was also difficult to detect these dipeptides in the semen of vasectomized patients, indicating that these dipeptides are not derived from the prostate, seminal vesicles, or other accessory gonads (data not shown).

We also found that DPEP3 was expressed in sperm and that levels of this dipeptide were reduced in varicocele patients. This will lead to a reduction in the concentrations of leucine, glutamic acid, tyrosine, phenylalanine, and isoleucine, in the seminal plasma. A reduction in the levels of leucine will result in a reduction in antioxidant and anti-inflammatory ability [22]. A reduction of glutamic acid will lead to a reduction in acid resistance, thus making sperm vulnerable to damage [23].

\subsection{Limitations}

The disadvantage of this study is that it is a single center, small sample study. Only the left grade III varicose veins are selected for the study. The selected biomarkers need to be further confirmed in large samples and different stages of disease development.

\section{Conclusion}

In this study, we first used UHLPC-QE-MS-untargeted metabolomics to detect metabolites in the seminal plasma from varicocele patients and patients receiving microsurgical varicocelectomy. Eight metabolites and three dipeptides were selected as potential biomarkers. Varicocele may influence semen quality by amino acid metabolism. Microsurgical varicocelectomy may improve semen quality by rebalancing dipeptide metabolism which has an antioxidant effect, and by regulating phospholipid and sphingolipid metabolism. Further studies are now needed to further elucidate the mechanisms underlying varicocele and how microsurgical varicocelectomy can improve semen quality.

\section{Declarations}


XZ had full access to all of the data used in this study and takes responsibility for the integrity of the data and the accuracy of the data analysis. XZ, QZ and YT were involved in study conception and design. All authors helped to acquire the data. XZ and WL analyzed and interpreted the data. CD and WL drafted the manuscript. CD and WL performed the statistical analysis. All authors helped to critically revise the manuscript for important intellectual content. XZ and QW obtained the funding. There were no administrative, technical or material support, and no supervisory arrangements to consider.

Financial disclosures: The authors declare no competing interests.

Funding/Support and role of the sponsor: This study was supported by the Natural Science Foundation of Guangdong Province to Xinzong Zhang [Grant No. 2114050002550], the Guangzhou Science and Technology Plan Project to Qiling Wang [Grant No. 202002030480], and the Innovation Team Project of Guangdong Institute of family planning science and technology to Xinzong Zhang [Grant No. C-01].

\section{CRediT authorship contribution statement:}

Xinzong Zhang: Conceptualization, Methodology, Formal analysis, Investigation, Data curation, Funding acquisition. Cuncan Deng: Investigation, Data curation, Writing - original draft, Visualization. Wujiang Liu: Conceptualization, Methodology, Formal analysis, Investigation, Data curation, Writing - original draft, Writing - review \& editing, Visualization. Huang Liu: Methodology, Software, Sample collection. Yu Zhou: Methodology, Software, Sample collection. Qianyi Li: Methodology, Software, Sample collection. Houbin Zheng: Methodology, Software, Sample collection. Qiling Wang: Data curation, Funding acquisition. Min Jiang: Data curation and data administration. Tao Pang: Sample collection and data management. Chunjie Ma: Sample collection and data management. Cheng Huang: Computer analysis, Software, Statistics. Qingguo Zhao: Resources. Yunge Tang: Conceptualization, Methodology, Formal analysis, Project administration.

\section{Appendix A. Supplementary data}

Correspondence and requests for materials should be addressed to X.Z.Z. or G.Y.T.

\section{Competing interests}

The authors declare no competing interests.

\section{Acknowledgements}

We would like to thank Dr Zhao Jiao for assisting us with the metabolomics analysis. We would also like to thank International Science Editing (http://www.internationalscienceediting.com) for editing this manuscript.

\section{References}


1. Baazeem, A. et al. Varicocele and male factor infertility treatment: a new meta-analysis and review of the role of varicocele repair. Eur Urol, 60 (4), 796-808 (2011).

2. Alsaikhan, B., Alrabeeah, K., Delouya, G. \& Zini, A. Epidemiology of varicocele. Asian J Androl, 18 (2), 179-181 (2016).

3. Kantartzi, P. D., Goulis, C. D., Goulis, G. D. \& Papadimas, I. Male infertility and varicocele: myths and reality. Hippokratia, 11 (3), 99-104 (2007).

4. Damsgaard, J. et al. Varicocele Is Associated with Impaired Semen Quality and Reproductive Hormone Levels: A Study of 7035 Healthy Young Men from Six European Countries. Eur Urol, 70 (6), 1019-1029 (2016).

5. Zümrütbaş, A. E., Gülpınar, Â., Mermerkaya, M., Süer, E. \& Yaman, Ã. The effect of varicocele on sperm morphology and DNA maturity: does acridine orange staining facilitate diagnosis? Turk J Urol, 39 (3), 165-169 (2013).

6. Madhusoodanan, V., Patel, P., Blachman-Braun, R. \& Ramasamy, R. Semen parameter improvements after microsurgical subinguinal varicocele repair are durable for more than 12 months. Can Urol Assoc J, 14 (3), E80-E83 (2020).

7. Birowo, P., Rahendra Wijaya, J., Atmoko, W. \& Rasyid, N. The effects of varicocelectomy on the DNA fragmentation index and other sperm parameters: a meta-analysis. Basic Clin Androl, 30, 15 (2020).

8. Sajadi, H. et al. Varicocelectomy May Improve Results for Sperm Retrieval and Pregnancy Rate in Non-Obstructive Azoospermic Men. Int J Fertil Steril, 12 (4), 303-305 (2019).

9. Silay, M. S. et al. Treatment of Varicocele in Children and Adolescents: A Systematic Review and Meta-analysis from the European Association of Urology/European Society for Paediatric Urology Guidelines Panel. Eur Urol, 75 (3), 448-461 (2019).

10. Abdel-Meguid, T. A., Al-Sayyad, A., Tayib, A. \& Farsi, H. M. Does varicocele repair improve male infertility? An evidence-based perspective from a randomized, controlled trial.

11. Eur Urol 2011; 59(3): 455 - 61.

12. Engel, K. M. et al. Metabolomic profiling reveals correlations between spermiogram parameters and the metabolites present in human spermatozoa and seminal plasma. PloS One, 14, e0211679 (2019).

13. Jayaraman, V. et al. Identification of biochemical differences between different forms of male infertility by nuclear magnetic resonance (NMR) spectroscopy. Journal of Assisted Reproduction and Genetics, 31, 1195-1204 (2014).

14. Zhang, J. et al. Metabolomic analysis reveals a unique urinary pattern in normozoospermic infertile men. Journal of Proteome Research, 13, 3088-3099 (2014).

15. Krzastek, S. C., Smith, R. P. \& Kovac, J. R. Future diagnostics in male infertility: genomics, epigenetics, metabolomics and proteomics. Transl Androl Urol, 9 (Suppl 2), S195-S205 (2020).

16. Ma, P. et al. Characterizing semen abnormality male infertility using non-targeted blood plasma metabolomics. PLoS One, 14 (7), e0219179 (2019). 
17. Mehrparavar, B., Minai-Tehrani, A., Arjmand, B. \& Gilany, K. Metabolomics of Male Infertility: A New Tool for Diagnostic Tests. J Reprod Infertil, 20 (2), 64-69 (2019).

18. Boguenet, M. et al. Metabolomic signature of the seminal plasma in men with severe oligoasthenospermia. Andrology, 8 (6), 1859-1866 (2020).

19. Murgia, F. et al. Seminal Fluid Metabolomic Markers of Oligozoospermic Infertility in Humans. Metabolites, 10 (2), 64 (2020).

20. Mumcu, A., Karaer, A., Dogan, B. \& Tuncay, G. Metabolomics analysis of seminal plasma in patients with idiopathic Oligoasthenoteratozoospermia using high-resolution NMR spectroscopy. Andrology, 8 (2), 450-456 (2020).

21. Mejia, E. M. \& Hatch, G. M. Mitochondrial phospholipids: role in mitochondrial function. $J$ Bioenerg Biomembr, 48 (2), 99-112 (2016).

22. Bartke, N. \& Hannun, Y. A. Bioactive sphingolipids: metabolism and function. J Lipid Res, 50 (Suppl(Suppl), S91-6 (2009).

23. Erukainure, O. L. et al. Oxidative testicular injury: effect of L-leucine on redox, cholinergic and purinergic dysfunctions, and dysregulated metabolic pathways., 53 (3), 359-380 (2021).

24. Holden, S. A. et al. Relationship between in vitro sperm functional assessments, seminal plasma composition, and field fertility after Al with either non-sorted or sex-sorted bull semen., 87, 221-228 (2017).

\section{Figures}


A

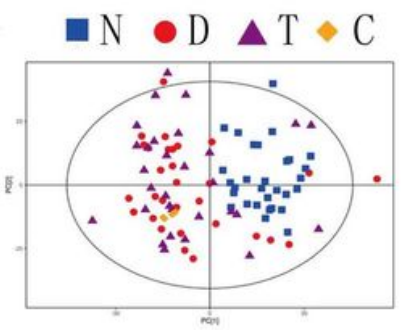

D

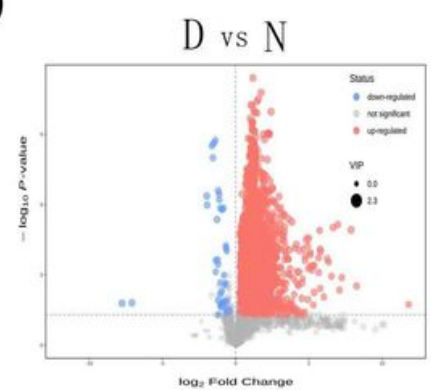

G

D vs $\mathrm{N}$

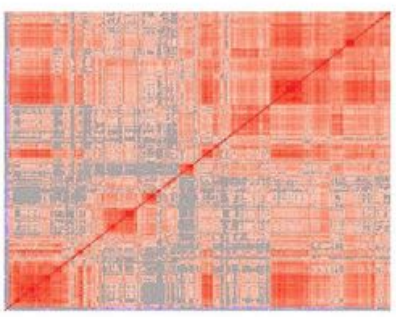

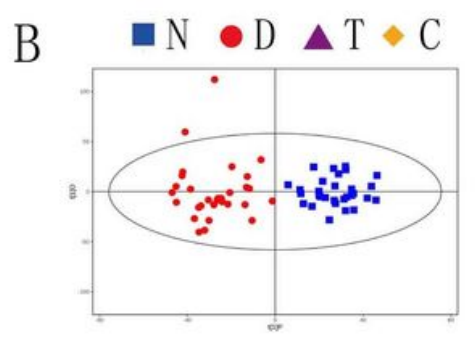

E

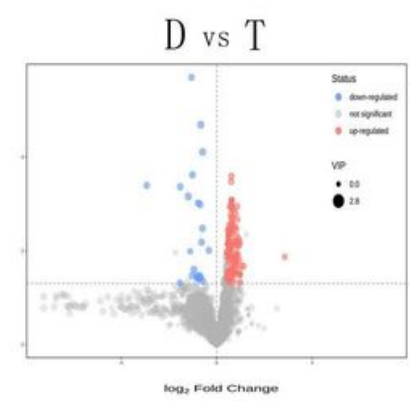

$\mathrm{H}$

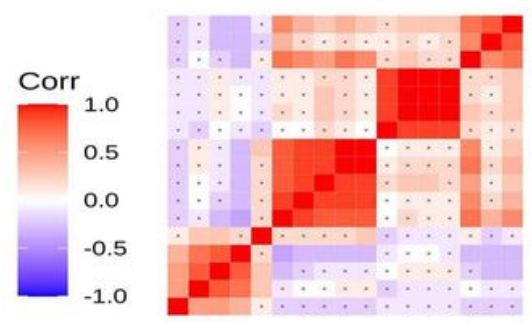

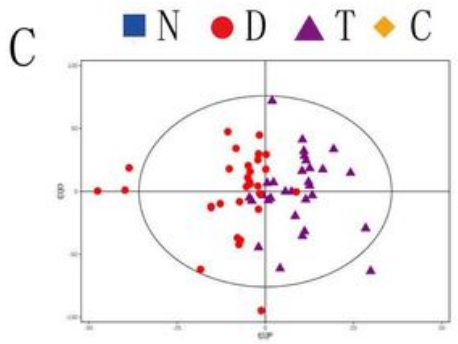

$\mathrm{F}$

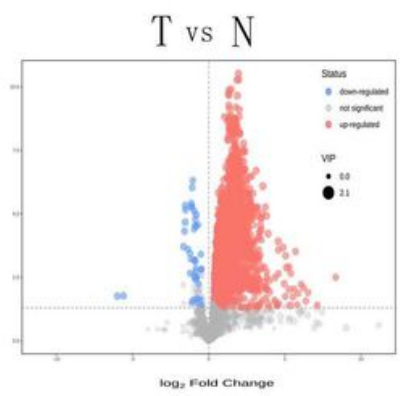

I

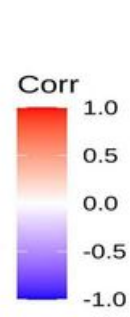

T vs $\mathrm{N}$

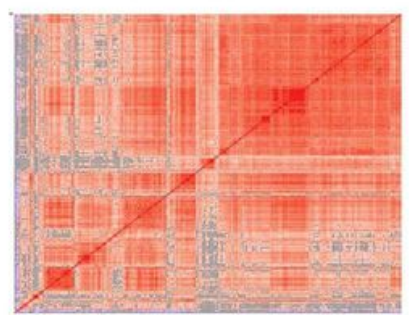

\section{Figure 1}

$A, B, C$ : Quality control of metabolomics analysis. Panel A shows a PCA(Principal Component Analysis)score scatter diagram for all samples (including the QC sample).The abscissa PC [1] and ordinate PC [2] represent the scores of the first and second principal components, respectively; the scatter color and shape represent the experimental groups of samples. Panels B and C show the OPLSDA(Orthogonal Partial Least Squares Discrimination Analysis) scatter chart. The abscissa t [1] P represents the predicted principal component score of the first principal component, the ordinate $t$ [1] 0 represents the orthogonal principal component score, and the scatter shape and color represent different experimental groups.N: normal control group, D: varicocele group, T: varicocelectomy group, C: quality control group. D,E,F: Differentially expressed metabolites represented in the form of volcano plots. Each point represents a metabolite. The abscissa represents multiple changes of the group compared with each substance (the logarithm based on 2), the ordinate represents the $p$ value from the Student's t-test (the negative logarithm was based on 10), and the scatter size represents the VIP value of the OPLS-DA mode. The larger the scatter, the greater the VIP value. The scatter color represents the final screening result. The significantly up-regulated metabolites are shown in red, the significantly down-regulated 
metabolites are shown in blue, and the metabolites without a significant difference are shown in gray. N: normal control group, D: varicocele group, T: varicocelectomy group. G,H,I: Correlation analysis of differentially expressed metabolites. The abscissa and ordinate represent a comparison of the differentially expressed metabolites between groups. The color blocks in different positions represent the correlation coefficient between the metabolites at corresponding positions: red indicates positive correlation, and blue indicates negative correlation. Insignificant correlation is shown as a cross. N: normal control group, D: varicocele group, T: varicocelectomy group.

D vs $\mathrm{N}$

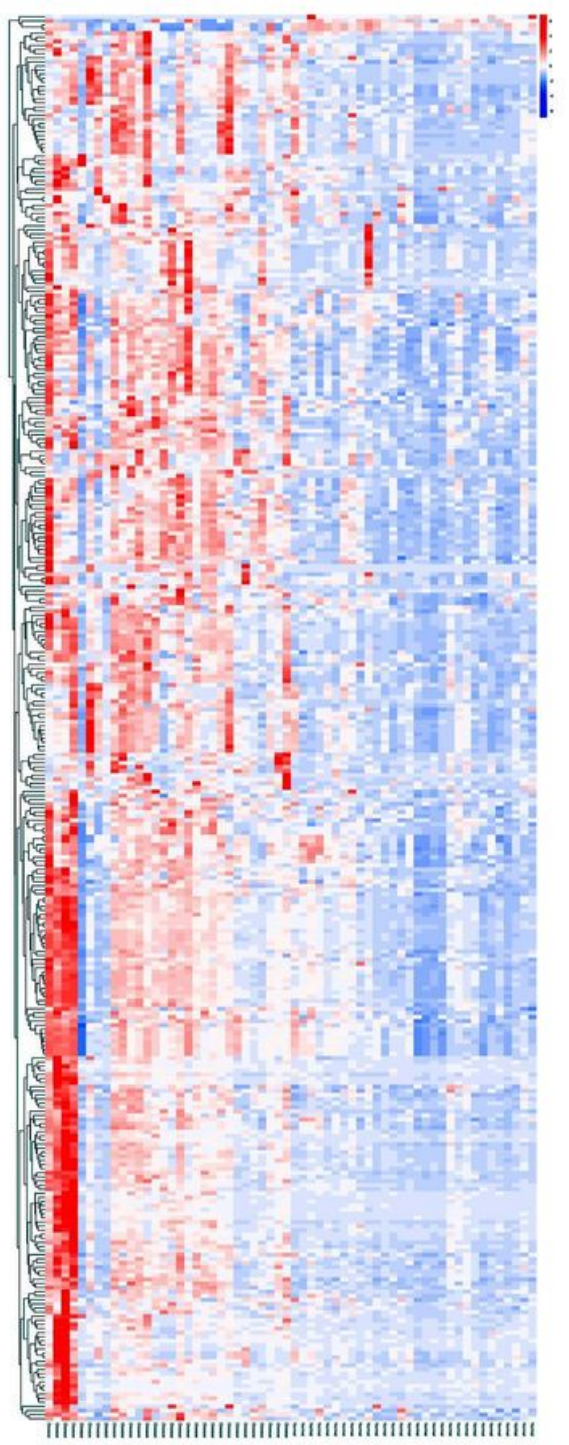

D vs T

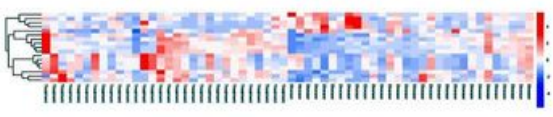

T vs $\mathrm{N}$

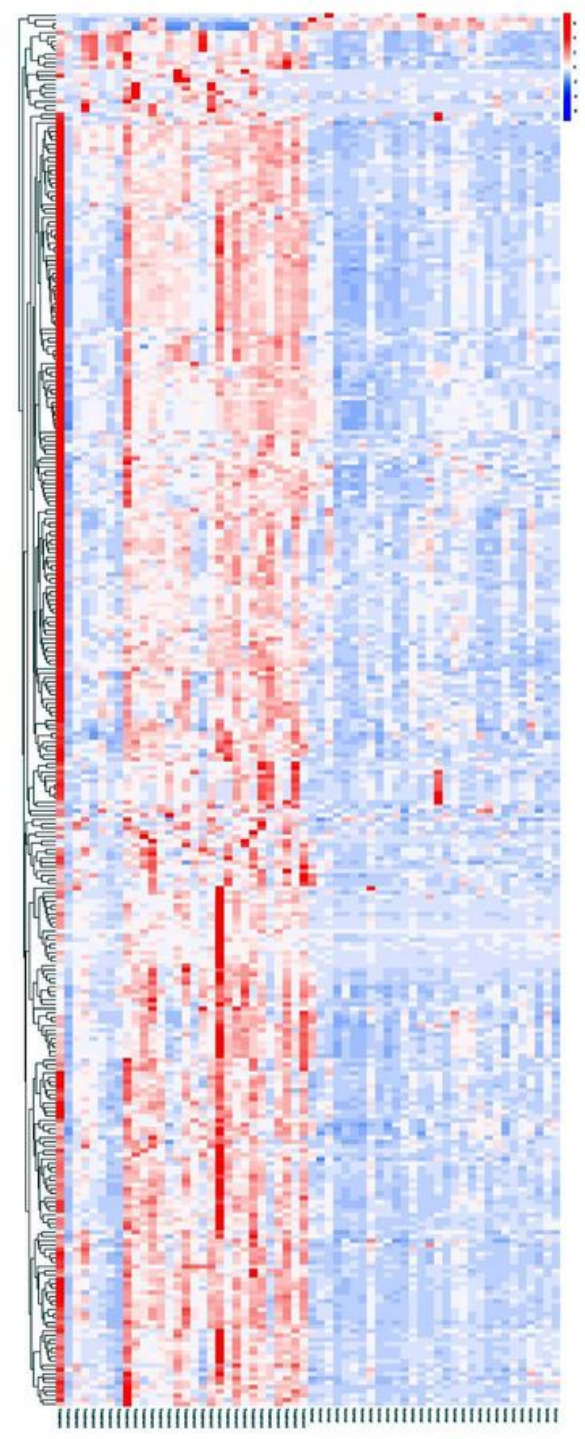

Figure 2

Heat map of hierarchical clustering analysis. The abscissa represents different experimental groups, the ordinate represents the differentially expressed metabolites by group, and the color blocks at different 
positions represent the relative expression of the corresponding metabolites. N: normal control group, D: varicocele group, T: varicocelectomy group.

A

A

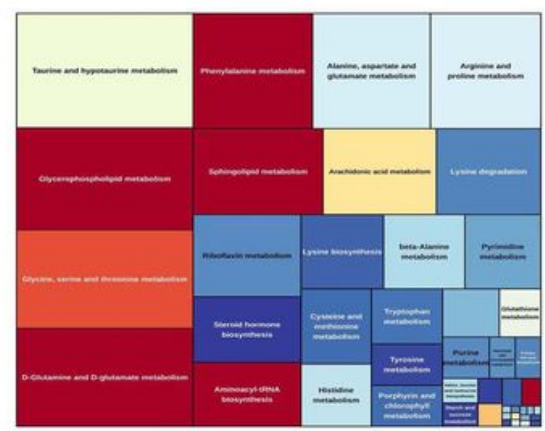

B

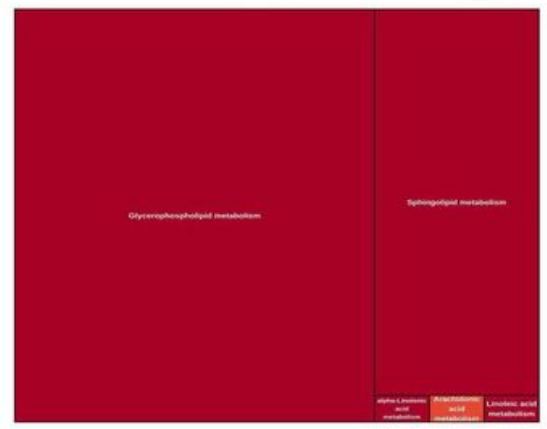

C

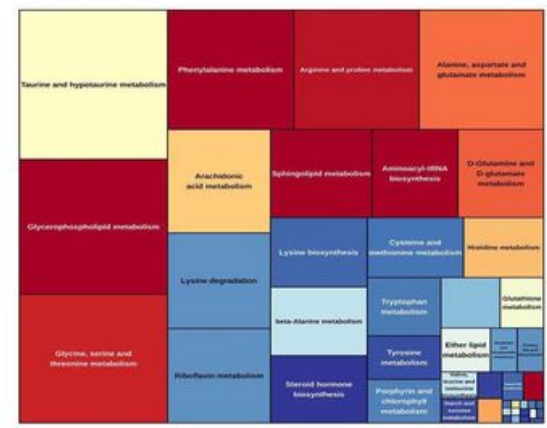

3. 0
$\mathrm{D}$

D vs N

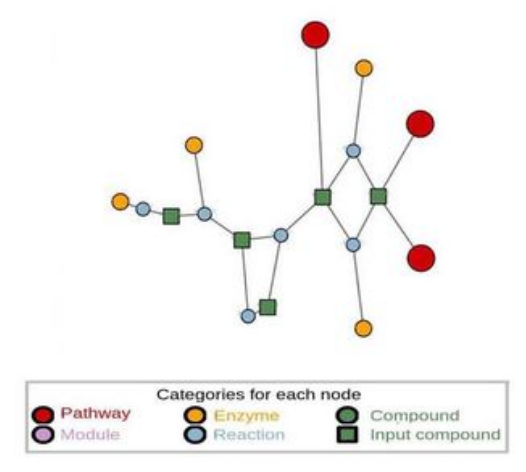

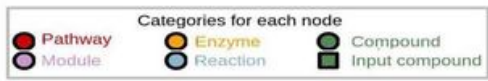

E

D vs T

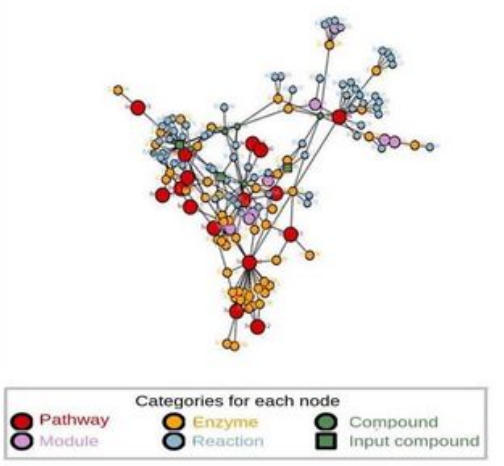

F

T vs $\mathrm{N}$

\section{Figure 3}

$A, B, C$ : Pathway analysis of different metabolites. The results of metabolic pathway analysis are shown as a rectangular tree. Each block in the rectangular tree represents a metabolic pathway, and the block size represents the influential factors of the pathway in the topological analysis. The larger the block size, the larger the influential factor; the block color represents the $p$ value of enrichment analysis (taking a negative natural logarithm, i.e. - In (P)). The darker the color, the smaller the $\mathrm{P}$ value, and the more significant the degree of enrichment. N: normal control group, D: varicocele group, $\mathrm{T}$ : varicocelectomy group. D,E,F: Regulatory network analysis of differentially expressed metabolites. The red dot represents a metabolic pathway, the yellow dot represents a substance related to a regulatory enzyme, the green dot represents the background material of a metabolic pathway, the purple dot represents the molecular module (class), the blue dot represents a chemical interaction reaction, and the green dot represents the differentially expressed substances. N: normal control group, D: varicocele group, T: varicocelectomy group. 

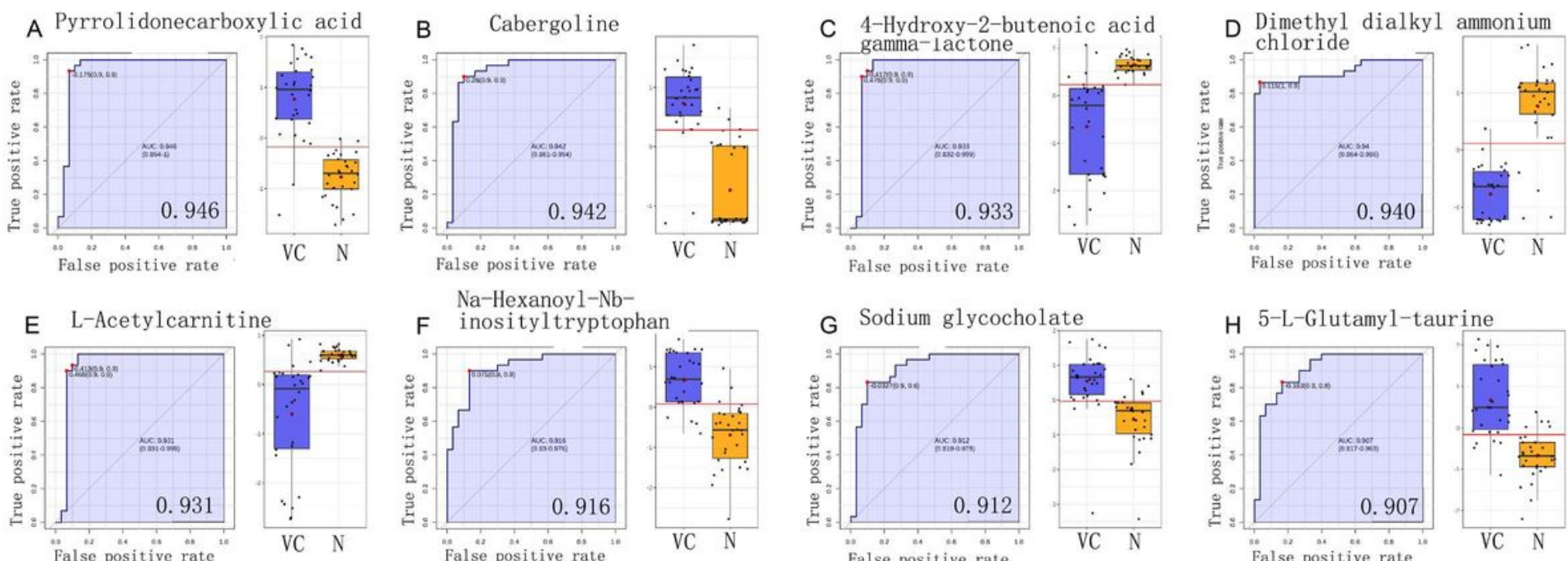

$\mathrm{Na}-\mathrm{Hexanoy} 1-\mathrm{Nb}-$
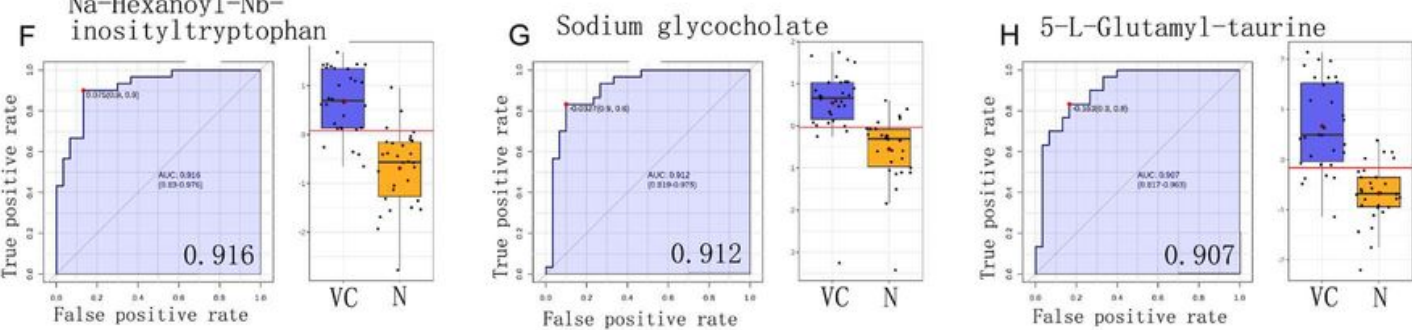

Figure 4

Potential biomarker analysis. The horizontal axis shows the false positive rate (=1-specificity). The vertical axis shows true positive rate (=sensitivity).AUC = area under curve; $\mathrm{N}$ : normal control group, $\mathrm{VC}$ : varicocele group.

A
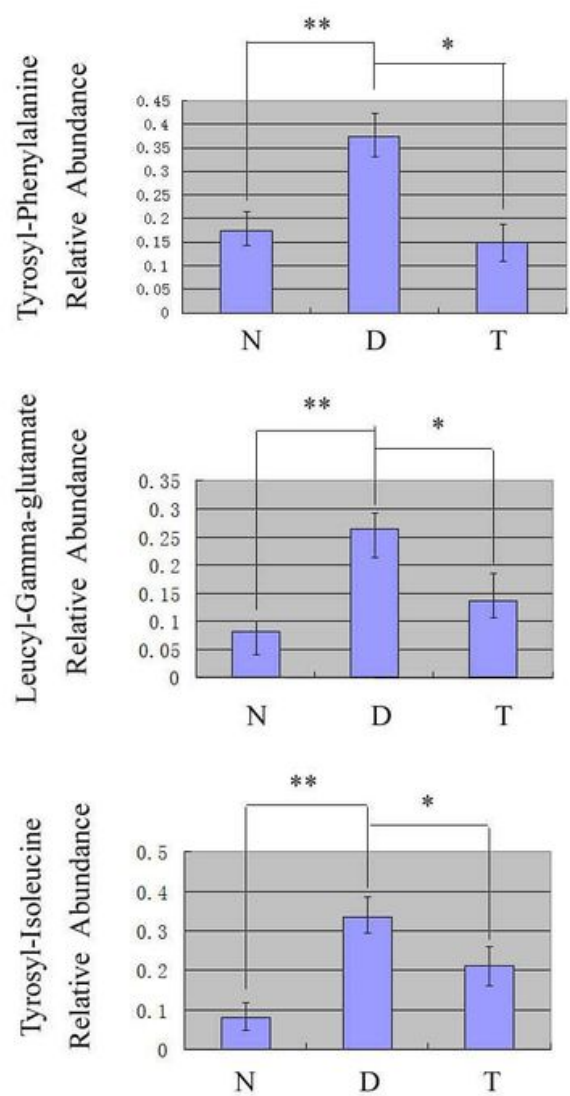

B

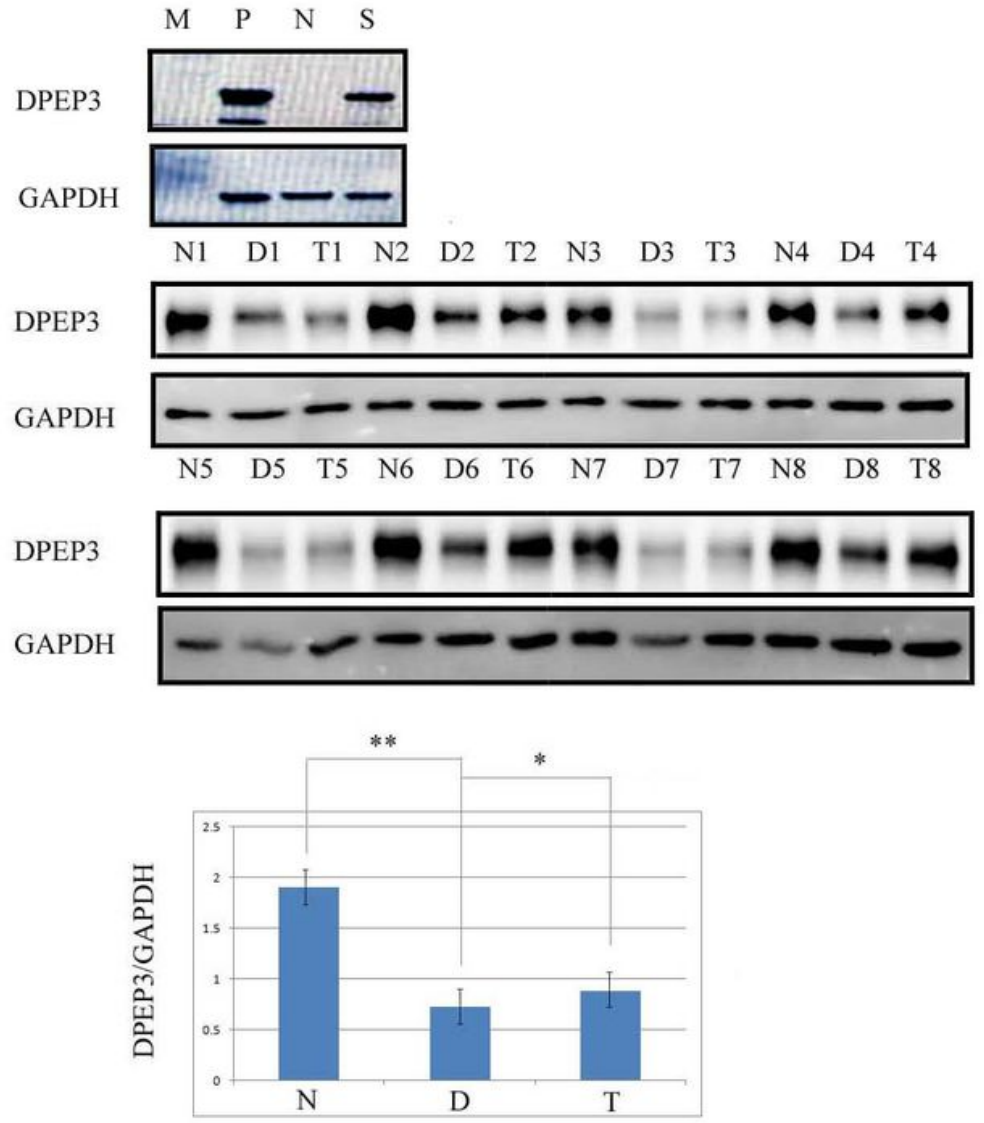

Figure 5 
Dipeptide metabolism of sperm and seminal plasma. Panel A shows the relative abundance of the three dipeptides confirmed by targeted metabonomics. Panel B shows representative western blots for DPEP3 expression among the normal control group, varicocele group and varicocelectomy group. Blots were distributed on three different gels. Electrophoresis, hybridization and exposure were performed under the same conditions. The intensity of the bands was analyzed by Image $\mathrm{J}$ software, and $\mathrm{t}$-test was used for statistical analysis. Panel $\mathrm{C}$ shows statistical results for each group. P: positive control, N: negative control, S: sample, N: normal control group, $\mathrm{D}$ : varicocele group, $\mathrm{T}$ : varicocelectomy group. ${ }^{\star \star} \mathrm{P}<0.01$; $* \mathrm{P}<0.05$.

\section{Supplementary Files}

This is a list of supplementary files associated with this preprint. Click to download.

- SupplementoryTable1/nclusionandexclusioncriteriaoftheparticipants.doc

- SupplementaryTable2Statisticalanalysisofimportantdifferentiallyexpressedmetabolites.doc

- Methodssection.doc 